\title{
Interpersonal Closeness and Social Reward Processing
}

\author{
Pascal Vrticka \\ Center for Interdiscipinary Brain Sciences Research, Department of Psychiatry and Behavioral Sciences, School of Medicine, Stanford University, Stanford, \\ California 94305-5795 \\ Review of Fareri et al.
}

According to the phylogenetic perspective of social engagement and attachment proposed by Porges (2003), human social behavior fundamentally depends on a dynamic "pushpull" mechanism between two opposing emotional neural circuits (Fig. 1). Within this framework, major importance is attributed to a social approach system promoting a sense of safety/security through close social interactions, sustained by reward-related brain activity. This is contrasted with more primitive survival-enhancing tendencies of a social aversion system, which operates through activation of sympathetic flight-or-fight circuits (Macdonald and Macdonald, 2010). Central to the proper functioning of the basic emotional module of positivity in human social behavior is the assumption that mutual social interactions are experienced as innately rewarding. Mounting evidence from functional magnetic resonance imaging (fMRI) experiments supports this notion by demonstrating increased activity in dopaminergic brain circuits not only during interactions with loved ones (children, parents, partners) or friends, but with any contextually relevant person with a cooperative relationship (e.g., engaged in a joint task) (Vrticka and Vuilleumier, 2012).

A recent article in The Journal of Neuroscience by Fareri and colleagues (2012)

Received July 3, 2012; revised July 23, 2012; accepted July 25, 2012.

This work was supported by a Swiss National Science Foundation Grant (No. 136480) to P.V. Many thanks to David Hong for very helpful comments on the manuscript.

Correspondence should be addressed to Dr. Pascal Vrticka, Center for Interdisciplinary Brain Sciences Research, Department of Psychiatry and Behavioral Sciences, School of Medicine, Stanford University, 401 Quarry Road, MC 5795, Stanford, CA 94305-5795. E-mail: pvrticka@stanford.edu. DOI:10.1523/JNEUROSCI.3157-12.2012

Copyright $\odot 2012$ the authors $\quad 0270-6474 / 12 / 3212649-02 \$ 15.00 / 0$ extends extant literature on the importance of reward-related signal processing within social settings. The authors show that blood oxygen level-dependent (BOLD) signal change in the ventral striatum and medial prefrontal cortex was significantly stronger when participants shared monetary gains with a friend than when rewards were shared with either an unknown confederate or a computer. Such findings bolster the notion that the reward centers of the human brain activate most strongly to a combination of positive personal and social information (Vrticka et al., 2008). These brain activation patterns were furthermore mirrored in high subjective ratings of excitement for shared positive outcomes with a friend, as well as high skin conductance responses during the same condition, reflecting increased biological arousal.

Reward-related responses to positive social interactions have previously been shown to strongly depend on individual differences in a specific measure related to interpersonal closeness, namely attachment avoidance (Vrticka and Vuilleumier, 2012). This psychological trait is marked by a high desire for independence and self-sufficiency, combined with a view of close relationships as unimportant (Mikulincer and Shaver, 2007). In one fMRI study, high attachment avoidance was found to negatively correlate with responses in reward-related brain areas (ventral striatum and orbitofrontal cortex) in social settings, namely in mothers viewing images of their own versus unknown babies (Strathearn et al., 2009). Another fMRI study demonstrated that high attachment avoidance was associated with low ventral striatum activation when participants obtained positive social feedback during successful trials in a perceptual game, whereas striatum activity representing subjective success (i.e., winning versus losing, per se) remained high and unaffected (Vrticka et al., 2008). Such data are further supported by results from a behavioral study revealing selectively decreased pleasantness ratings of positive social (but not positive nonsocial) visual scenes (Vrticka et al., 2012). Together, these findings suggest that a high avoidant attachment style not only diminishes basic reward responses to social stimuli normally perceived as highly positive, but also strongly modulates how personal success is integrated with social information components.

The results reported by Fareri and colleagues (2012) importantly extend such knowledge of how interpersonal closeness could modulate reward-related responses during positive social interactions. The authors applied the Inclusion of the Self in the Other Scale (IOS) (Aron et al., 1992), which assesses the "feeling close" and "behaving close" aspects of social interactions, and had their participants rate the closeness of their relationship with the friend also participating in the study. For participants scoring high on the IOS, ventral striatum activity was found to be higher for comparisons of "friend versus confederate" and "friend versus computer." However, no such differences were observed in low IOS participants. Even more intriguing was the finding that activity in low IOS participants was nonspecifically increased during monetary gains (versus losses) across all three conditions (friend, confederate, computer). This suggests that low IOS participants were 

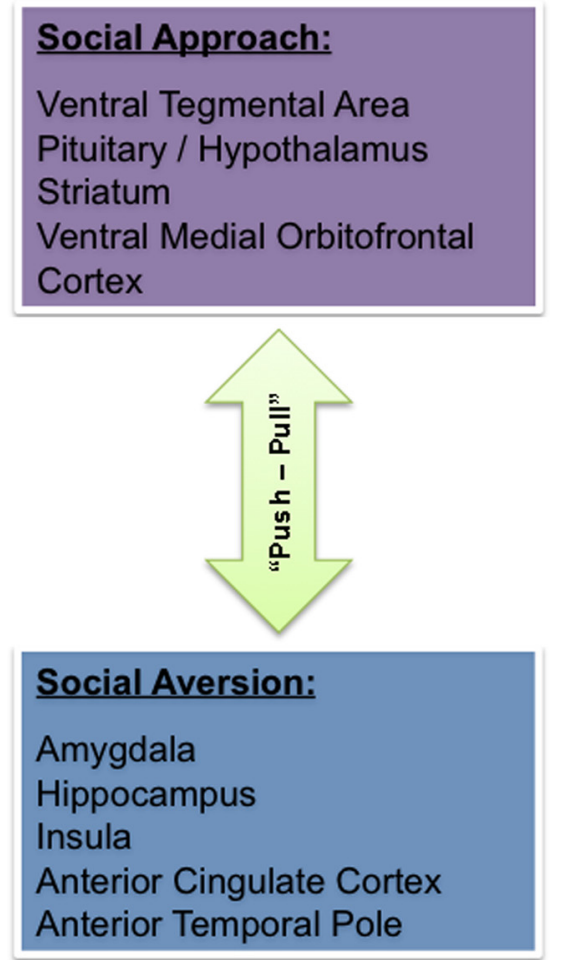

\section{AFFECTIVE PROCESSING}

Figure 1. Illustration of the dynamic "push-pull" between social approach and aversion in the affective processing module of human social interaction (derived from Vrticka and Vuilleumier, 2012). According to the phylogenetic perspective of social engagement and attachment proposed by Porges (2003), human social functioning is determined by two opposite emotional brain systems representing positive (social approach; purple) versus negative (social aversion; blue) information. Whereas the social approach module mainly includes dopaminergic pathways (ventral tegmental area, striatum, ventral medial orbitofrontal cortex), as well as the pituitary/hypothalamus as the main site of oxytocin synthesis, the social aversion module operates through brain areas involved in fear/threat (amygdala), stress (hippocampus), disgust/empathy for pain/social rejection (insula and anterior cingulate cortex), and sadness (anterior temporal pole).

mainly focusing on the personally relevant aspect of winning, rather than the social aspect of sharing. As mentioned above, sharing with a friend is expected to be experienced as more socially rewarding than sharing with an unknown confederate or a computer, which was observed in high IOS participants. Consequently, participants with low IOS scores appeared to show a lack of social context integration during personally rewarding experiences.

Although no investigation reported to date directly compares attachment avoid- ance and IOS, such an approach nonetheless appears sound and valid. This comes from the fact that attachment avoidance is characterized by the major relational goal of wanting less closeness, as reflected by many items of attachment self-report instruments such as, "I am uncomfortable getting too close to others" (for a list of available attachment questionnaires and included single items, see Mikulincer and Shaver, 2007). Furthermore, there is indirect evidence that individuals oriented toward avoidant attachment are especially sensitive to situational and relational cues related to wanting less closeness (Mashek et al., 2011). This nicely matches the initial definition of relationship closeness applied by the IOS (Aron et al., 1992). Accordingly, the available neuroscientific data regarding the influence of individual differences in low interpersonal closeness on reward-related brain activity during positive social interactions suggests the following. In people scoring high on interpersonal closeness (high IOS/low attachment avoidance), reward-related activity is selectively increased in positive social versus nonsocial settings, reflecting a proper representation of the intrinsically positive value of mutual social interactions, as well as an intact integration of personal and social information components (Vrticka and Vuilleumier, 2012). In contrast, individuals with low interpersonal closeness indexes (low IOS/high attachment avoidance) display either strongly decreased reward-related processing of social positive information (Vrticka et al., 2008; Strathearn et al., 2009) or nonspecifically high BOLD signal in reward areas to both social and nonsocial positive stimuli, reflecting a strong bias toward personal/subjective versus social processing of information, i.e., winning versus sharing (Fareri et al., 2012). Put differently, low interpersonal closeness seems to sustain or overemphasize nonsocial positive reward representation while decreasing sensitivity to social positive reward encoding in different social contexts.

As outlined at the beginning of this article, social reward processing appears to represent a central emotional neurobiological component underlying human social behavior (social approach module), counteracting more primitive survivalenhancing social aversion mechanisms (Fig. 1) (Porges, 2003). Within this framework, mounting evidence implies that the social approach aspect of the dynamic emotional "push-pull" is susceptible to individual differences in perceived interpersonal closeness as reflected by low IOS and/or high attachment avoidance scores. Such effects could importantly modulate emotional homeostasis during social interactions, making affected individuals more prone to developing psychopathological conditions marked by social disturbance. Future investigations should therefore closely examine the neural substrates of the dynamic balance between social approach and aversion not only in healthy adults, as performed to date, but also within clinical populations. This could ultimately lead to the emergence of new and more specific intervention strategies of psychopathological conditions involving disturbed social functioning.

\section{References}

Aron A, Aron EN, Smollan D (1992) Inclusion of the other in the self scale and the structure of interpersonal closeness. J Pers Soc Psychol 63:596-612.

Fareri DS, Niznikiewicz MA, Lee VK, Delgado MR (2012) Social network modulation of reward-related signals. J Neurosci 32:90459052. 10.1523/JNEUROSCI.0610-12.2012.

Macdonald K, Macdonald TM (2010) The peptide that binds: a systematic review of oxytocin and its prosocial effects in humans. Harv Rev Psychiatry 18:1-21.

Mashek D, Le B, Israel K, Aron A (2011) Wanting less closeness in romantic relationships. Basic Appl Soc Psychol 33:333-345. 10.1080/ 01973533.2011.614164.

Mikulincer M, Shaver PR (2007) Attachment in adulthood: structure, dynamics, and change. New York: Guilford.

Porges SW (2003) Social engagement and attachment: A phylogenetic perspective. Ann N Y Acad Sci 1008:31-47. doi:10.1196/annals.1301.004.

Strathearn L, Fonagy P, Amico J, Montague PR (2009) Adult attachment predicts maternal brain and oxytocin response to infant cues. Neuropsychopharmacology 34:26552666.

Vrticka P, Vuilleumier P (2012) Neuroscience of human social interactions and adult attachment style. Front Hum Neurosci 6:212.

Vrticka P, Andersson F, Grandjean D, Sander D, Vuilleumier P (2008) Individual attachment style modulates human amygdala and striatum activation during social appraisal. PloS One 3:e2868.

Vrticka P, Sander D, Vuilleumier P (2012) Influence of adult attachment style on the perception of social and non-social emotional scenes. J Soc Pers Relations 29:530-544. 